Int. J. Dev. Biol. 49: 859-865 (2005)

doi: $10.1387 / \mathrm{ijdb} .051996 \mathrm{as}$

Original Article

\title{
Changes in E2F5 intracellular localization in mouse and human choroid plexus epithelium with development
}

\author{
ADAM SWETLOFF and PATRIZIA FERRETTI* \\ Developmental Biology Unit, Institute of Child Health, UCL, London, UK
}

\begin{abstract}
The choroid plexus epithelium (CPe) is a specialized epithelium involved primarily in the production of cerebrospoinal fluid (CSF) which is important for maintaining an optimal homeostatic environment for the brain. Although, the physiology of the $\mathrm{CPe}$ is fairly well understood, its development has not been thoroughly studied. It has been recently shown that mice lacking functional transcription factors, E2F5, foxJ1 or p73, develop non-obstructive hydrocephalus likely due to $\mathrm{CPe}$ dysfunction. We have further studied their expression in the mouse and human developing $\mathrm{CPe}$, focusing particularly on E2F5. We show here that in the mouse E2F5, foxJ1 and p73 transcripts are detectable as soon as the choroid plexuses form. E2F5 protein is also detected as soon as the choroid plexuses are morphologically apparent both in mouse and human, suggesting that its expression is regulated at the transcriptional level. E2F5 protein is down-regulated late in embryogenesis and this coincides with a change in its intracellular localization, from predominantly nuclear to cytoplasmic. The pattern of expression and intracellular localization of E2F5 in vivo does not appear to correlate with that of proliferating CPe cells, as indicated by protein cell nuclear antigen (PCNA) staining, but rather with their maturation, as changes in E2F5 localization from the nucleus to the cytoplasm parallel the morphological change from pseudostratified to cuboidal epithelium.
\end{abstract}

KEY WORDS: choroid plexus epithelium, E2F5, hydrocephalus, human, mouse, proliferation

\section{Introduction}

The choroid plexuses are secretory organs located within the ventricular system of the brain and forms the blood-cerebrospinal fluid (CSF) barrier. Adult choroid plexuses are villous structure that extend from the ependymal surface of the brain into the ventricular lumen. The choroid plexus consists of a single-layered cuboidal epithelium $(\mathrm{CPe})$ surrounding a highly vascular mesenchymal core. There are four choroid plexuses in the brain: one in each lateral ventricle of the cerebral hemispheres, one in the roof of the III ventricle and one in the caudal roof of the IV ventricle. In both mice and humans the IV ventricle choroid plexus is the first one to develop, between embryonic day 10.5 (E10.5) and E11.5 in the mouse and around 41 days of gestation (dg) in humans (Shuangshoti and Netsky, 1966). The lateral choroid plexuses are first identifiable at E12.5 in mouse and around 44dg in human embryos, whereas the III ventricle choroid plexuses are observed at $\mathrm{E} 14.5$ and $57 \mathrm{dg}$, respectively. The $\mathrm{CPe}$ originates from the neural tube, whereas its mesenchymal core originates from the paraxial mesenchyme and possibly, in the case of lateral ventricle choroid plexus, from the neural crest (Catala, 1998; Couly et al., 1993; Wilting and Christ, 1989). The CPe is involved in production and secretion of the CSF and therefore plays a key role in maintaining brain homeostasis. Defective circulation, reabsorption or production of CSF can lead to hydrocephalus, an accumulation of CSF in the ventricular system, that can affect brain function. Recently, occurrence of non-obstructive hydrocephalus has been reported in mice lacking functional transcription factors such as E2F5, foxJ1, foxc1 and p73 (Chen et al., 1998; Kume et al., 1998; Lindeman et al., 1998; Yang et al., 2000). In the case of foxJ1, a member of the forkhead-box (Fox)/winged helix gene family, the ventricular dilation found in $50 \%$ of the homozygous mutant mice is thought to be caused by defective CSF dynamics. This is likely due to the absence of cilia that, as in other ciliated epithelia of the body, are not present in the CPe of these mutants (Chen et al., 1998). Another member of the Fox gene family, foxc1, is mutated in the congenital hydrocephalus mouse and defective development of the arachnoid epithelium, that is impor-

Abbreviations used in this paper: $\mathrm{BCA}$, bicinchroninic acid; $\mathrm{CPe}$, choroid plexus epithelium; CSF, cerebrospinal fluid; dg, days of gestation; E, embryonic day; PBS, phosphate-buffered saline; PCNA, proliferating cell nuclear antigen; PVDF, polyvinylidene difluoride; RT-PCR, reverse transcription polymerase chain reaction; TTR, transthyretin.

\footnotetext{
*Address correspondence to: Dr. Patrizia Ferretti. Developmental Biology Unit, Institute of Child Health, 30 Guilford Street, London WC1N 1EH, UK. Fax: +44-20-7831-4366. e-mail: ferretti@ich.ucl.ac.uk
} 
tant for CSF absorption and expresses Foxc1, may underlie the hydrocephalic phenotype in these mice (Kume et al., 1998). Mice lacking functional p73, a member of the p53 family, that is expressed in the mouse $\mathrm{CPe}$ at E12.5, exhibit a progressive hydrocephalus as well as other defects such as hippocampal dysgenesis, chronic infections and inflammation and abnormalities in pheromone sensory pathways (Yang et al., 2000). p73 has been reported to regulate the expression of the water transporter aquoporin 3 (Zheng and Chen, 2001) and it is conceivable it may regulate members of this water transporter gene family expressed in the choroid plexus. Therefore it was suggested that p73 might affect either production or reabsorption of CSF. The case of hydrocephalus most convincingly due to increased CSF secretion is that observed in E2F5 knockout mice (Lindeman et al., 1998). These mice begin to suffer from a non-obstructive type of hydrocephalus 3-4 weeks after birth and do not show any other obvious developmental defect. Their CPe contains more electron-lucent cells, indicative of high secretory activity, than wild type mice. E2F5 belongs to a family (E2F1-E2F6) of transcriptional regulators involved in cell cycle regulation, but it does not seem itself necessarily involved in cell proliferation, at least in cultured fibroblasts from E2F5 mice (Lindeman et al., 1998; Gaubatz, et al., 2000). E2F5, foxj1 and p73 expression throughout choroid plexus development in the mouse has not been thoroughly examined and most information available on E2F5 expression is limited to mRNA analysis with no distinction between different choroid plexuses. We have therefore examined their expression patterns in the choroid plexuses of the IV and lateral ventricles during development by immunohistochemistry and Western blotting and by RT-PCR. We have also studied the E2F5 protein in the developing human choroid plexuses to establish whether its pattern of expression is conserved across species and they may therefore play a role in the pathology of congenital forms of hydrocephalus. We show here that foxj1, p73 and E2F5 are expressed early in the choroid plexuses and their expression is maintained throughout development, though E2F5 expression seems to decrease at late embryonic stages and its intracellular localization changes with CPe development. Expression of E2F5 in the mouse has also been studied in parallel with that of the proliferating cell nuclear antigen, PCNA, that localizes to the nucleus during the $\mathrm{G} 1$ to $S$ phase of the cell cycle, in order to investigate the relationship between E2F5 expression and cell proliferation. We have not observed any obvious correlation in the expression of these two proteins linking E2F5 to proliferation of the $\mathrm{CPe}$, but have found that the intracellular localization of E2F5 is developmentally-regulated.

\section{Results}

\section{mRNA expression in the mouse choroid plexus}

The expression of E2F5, foxJ1 and p73 mRNA was studied by RT-PCR in the developing IVth ventricle choroid plexus of E12.5, E16.5, E17.5 and adult mice (Fig. 1A). All three genes were expressed at early stages of choroid plexus development and could be detected throughout development and in the adult choroid plexus, like transthyretin (TTR), a thyroid hormone binding protein that in the CNS is a specific marker for the CPe. The E2F5 transcript, however, seemed to slightly decline with development and Fox $\mathrm{J} 1$ expression appeared consistently higher at
A

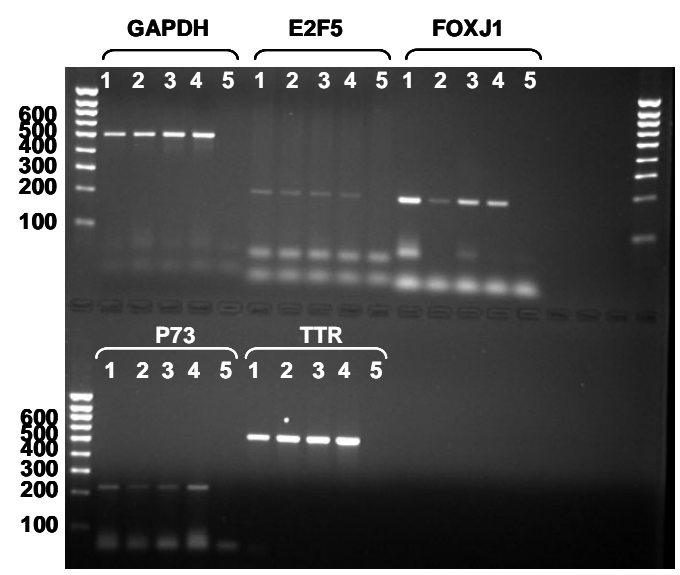

B

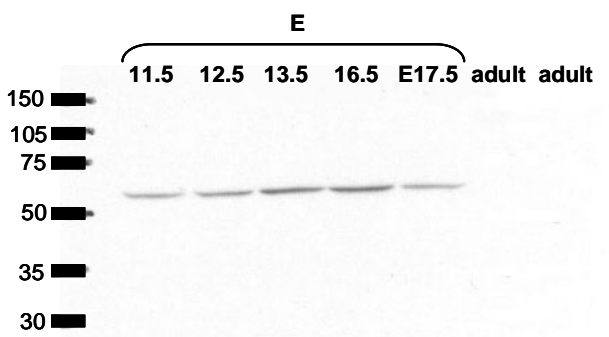

C E
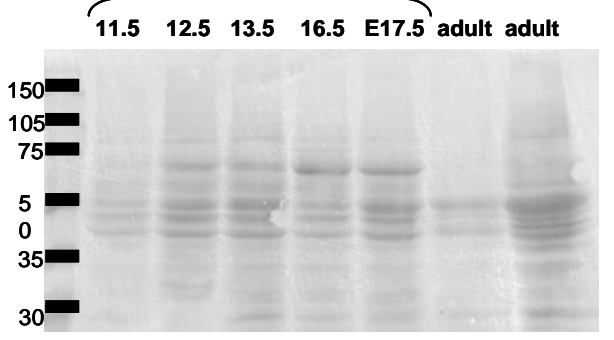

Fig. 1. Analysis of E2F5, FoxJ1 and p73 mRNA in the developing and adult mouse IV ventricle choroid plexus by RT-PCR and of E2F5 protein in the developing brain by Western blotting. Whole choroid plexus RNA was prepared from (1) E12.5, (2) E16.5, (3) E17.5 and (4) adult mice. Water was used as negative control (5). Expected product sizes are: GAPDH (491 bp), E2F5 (227 bp), foxJ1 (205 bp), p73 (232 bp), TTR (505 bp). The DNA molecular weight ladder is on the left. (B) Detection of E2F5 in whole brain protein extracts from different developmental stages (E11.517.5 and adult). The molecular weight of the E2F5 protein is $\sim 54 \mathrm{kDa}$. (C) Corresponding Western blot membrane stained with Ponceau red after transfer showing that equivalent amounts of protein were loaded for each sample.

E12.5 than at any other stage examined following normalization with GAPDH (not shown). No major changes in p73 expression were apparent.

E2F5 and proliferation in mouse and human choroid plexus

Expression of E2F5 protein was studied both by Western blotting and immunohistochemistry. Western blot analysis of protein extracts from mouse brain at different stages of development showed that the antibody used detected a single band of approximately $54 \mathrm{kDa}$ in embryonic brains at all stages examined. 
This molecular weight is consistent with that previously reported (Vaishnav et al., 1998). A decrease in E2F5 expression in the developing brain was observed at E17.5 and the protein was hardly detectable in the adult brain (Fig. 1B-C).

To specifically assess expression of E2F5 in the choroid plexus this antibody was used to stain paraffin sections of mouse and human developing brains. In E11.5 mice, strong nuclear expression of the E2F5 protein was observed in the neuroepithelium and in the epithelium of the choroid plexus primordia (Fig. 2A). In contrast, the choroid plexus mesenchyme was largely negative. E2F5 staining was abolished by preabsorption with the blocking peptide (Fig. 2B). By E13.5, staining was observed throughout the brain but was stronger in the periventricular regions (not shown). Both IVth and lateral ventricle choroid plexuses were intensely positive (Fig. 2C-D). A predominantly nuclear intense staining was detected in the $\mathrm{CPe}$, whereas a faint cytoplasmic staining was observed in the choroid plexus mesenchyme. By E17.5, the levels of staining in the neuroepithelium and CPe were markedly reduced and had become predominantly cytoplasmic (Fig. 2E). In the brain parenchyme, only a few areas, mainly in the ventricular and subventricular region, were still E2F5-positive. In contrast, staining of the whisker barrels was intensely positive and nuclear (not shown). Differences in E2F5 intracellular localization were clearly observed also in CPe cells in vitro, with the protein being localized to the nucleus in low density cultures and largely confined to the cytoplasm in denser, but still growing, cultures (Fig. 2F-G). In order to assess whether translocation of the E2F5 protein to the nucleus was associated with the proliferative state of the cells in vivo, we carried out a parallel analysis of E2F5 and PCNA distribution in the choroid plexus. Extensive PCNA staining was present in the neuroepithelium at E12.5 (Fig. $3 \mathrm{~A}$ ), but in the E11.5 choroid plexus primordium (not shown) and in the E12.5 IVth ventricle $\mathrm{CPe}$, PCNA staining was largely restricted to the stalk of the CPe (Fig. 3B) In contrast, strong E2F5 reactivity was present throughout the $\mathrm{CPe}$ (Fig. 3C). At E12,5, the lateral ventricle appeared to contain PCNA-positive cells not only at the stalk of the $\mathrm{CPe}$, but scattered throughout the epithelium (Fig. 3D). By E17.5 PCNA expression was restricted to the choroid plexus stalk in both IVth and lateral ventricles (Fig. 3E, G), whereas E2F5 was expressed not only in the stalk of the choroid plexus, but throughout the CPe cytoplasm (Fig. 3F). Unlike in the choroid plexus, E2F5 and PCNA expression overlapped in the cells lining the cerebellum and to some extent in the neuroepithelium. In the developing human brain, nuclear staining for E2F5 protein was found in the neuroepithelium surrounding the telencephalic vesicles as well as in the choroid plexus primordia budding out into the ventricular cavity at $44 \mathrm{dg}$ (Fig. $4 \mathrm{~A}$ ). At $52 \mathrm{dg}$, strong staining for
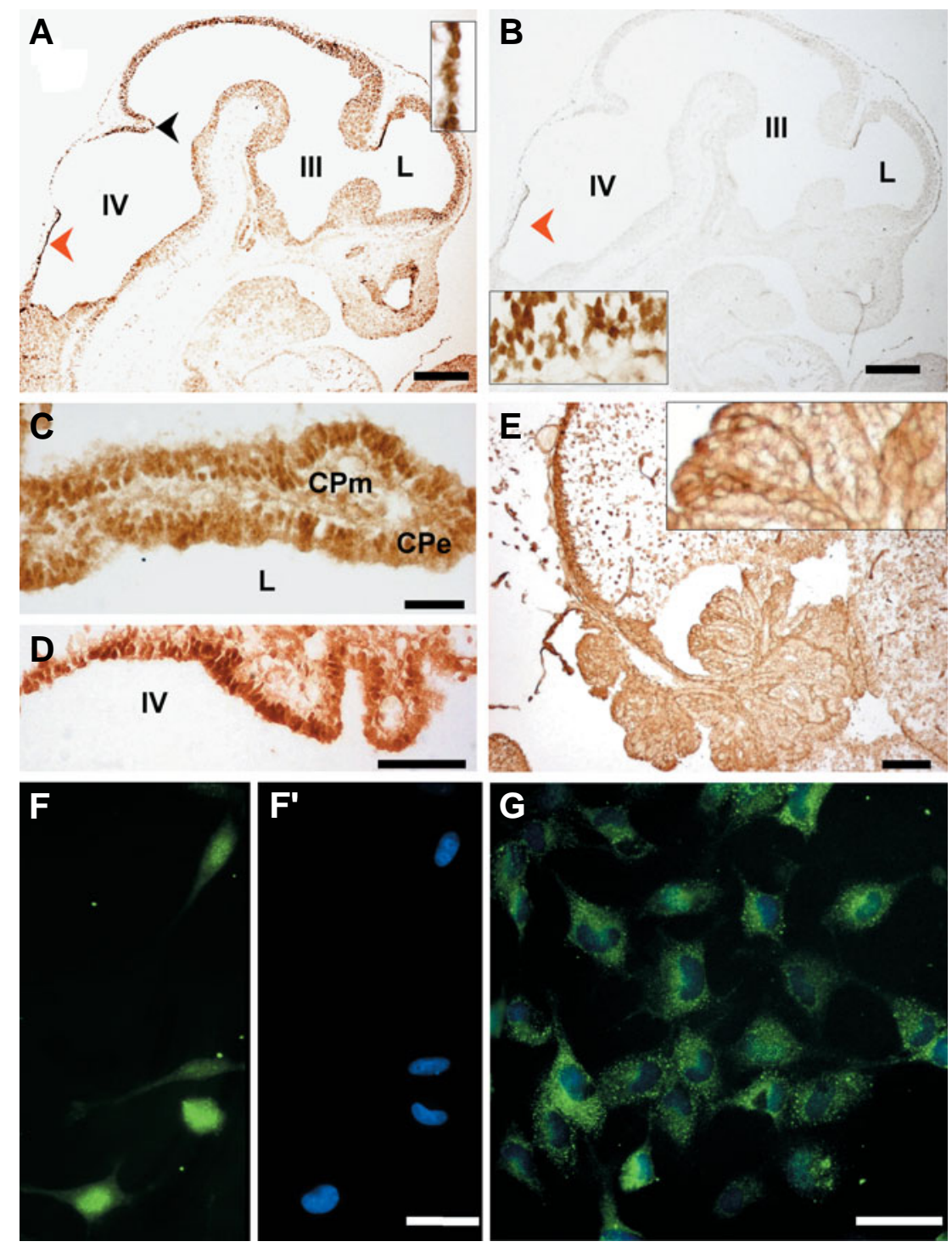

Fig. 2. E2F5 protein expression in the developing mouse choroid plexus (CP, brown) and in a choroid plexus cell line (green). (A) Sagittal view of an E11.5 mouse head showing E2F5 nuclear staining in the developing brain. Note positive staining in the presumptive IVth ventricle choroid plexus (red arrowhead and insert). The black arrowhead indicates the cerebellum primordium that is shown at higher magnification in the insert in (B) to better visualize the nuclear staining. (B) Adjacent section to that shown in (A) incubated with anti-E2F5 antibody pre-absorbed with the blocking peptide; no reactivity is observed. (C) Lateral and (D) IV ventricle choroid plexus at E13.5. Note the predominantly nuclear staining in both choroid plexuses. (E) E17.5 IV ventricle choroid plexus (high magnification in insert). Note that E2F5 staining is much lower than at E13.5 and largely cytoplasmic. (F-F') Nuclear E2F5 staining (green) can be observed in some cells in sparse TRCSFB-2 cultures; Hoechst nuclear staining is blue. (G) E2F5 staining is predominantly cytoplasmic in denser cultures. $\mathrm{CPe}$, choroid plexus epithelium; CPm, choroid plexus mesenchyme; III, III ventricle; IV, IV ventricle; L, lateral ventricle. Scale bars: $400 \mu \mathrm{m}$ in A$B, 25 \mu \mathrm{m}$ in $C$ and $50 \mu \mathrm{m}$ in $D-G$.

E2F5 was found in the neuroepithelium as well as in the choroid plexus of the telencephalic ventricle (Fig. 4B). The pseudostratified $\mathrm{CPe}$ exhibited strong nuclear staining and reactivity was observed also in the cytoplasm (Fig. 4C); intense nuclear labelling was present in the neuroepithelium. The choroid plexus mesen- 

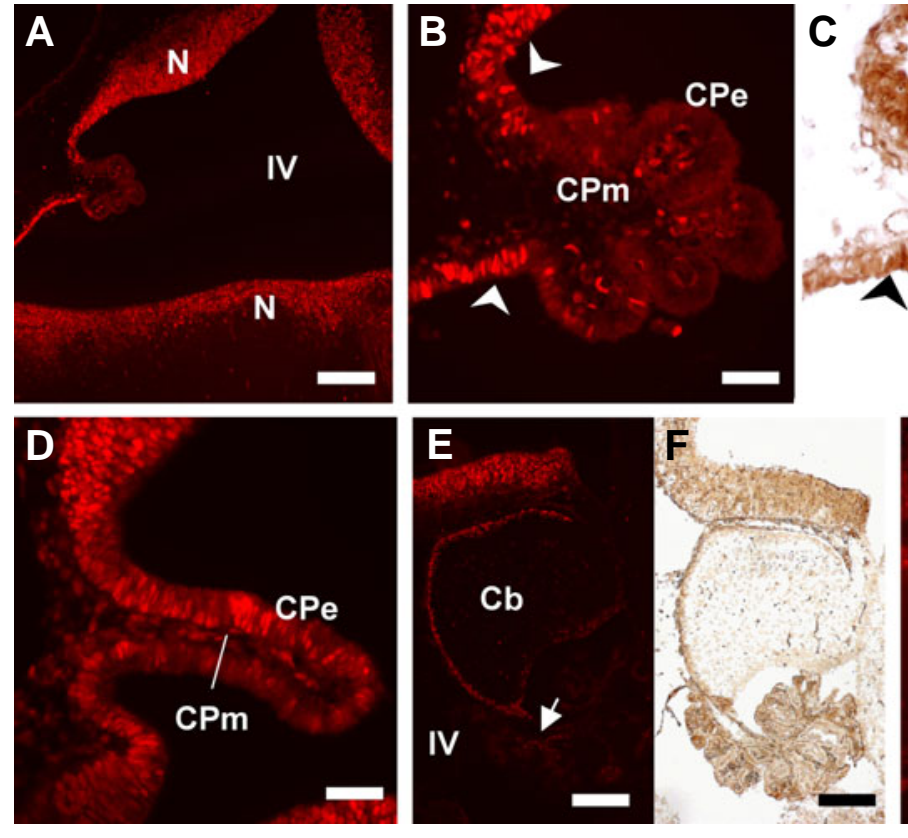
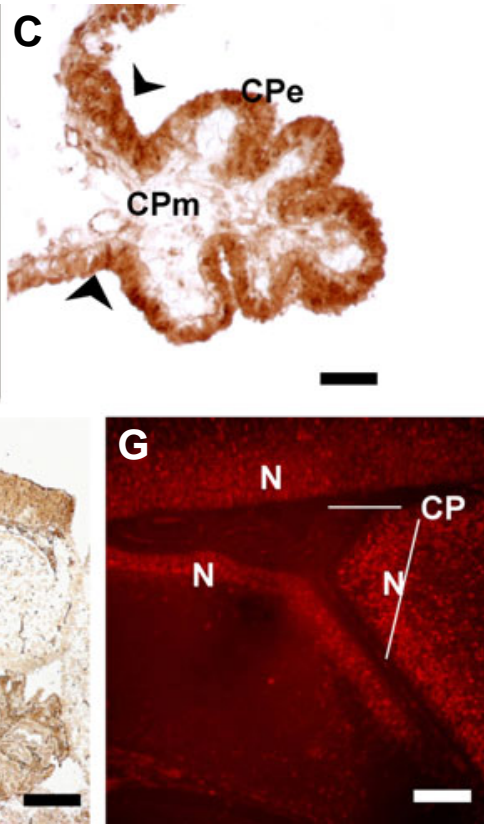

Fig. 3. PCNA and E2F5 protein localization in the mouse embryonic CPe. (A) E12.5 mouse IVth ventricle choroid plexus labelled for PCNA; staining is only localized to the root of the $\mathrm{CPe}$, whereas much staining is observed in the neuroepithelium. (B) High magnification of E12.5 mouse IVth ventricle stained for PCNA; note the positive staining at the stalk of the choroid plexus (arrowheads). (C) Adjacent section to that shown in (B) stained for E2F5 shows extensive reactivity in the $C P$. (D) E12.5 lateral ventricle stained for PCNA. Note positive nuclei throughout the $\mathrm{CPe}$. (E-F) E17.5 IVth ventricle choroid plexus stained for PCNA and E2F5, respectively. Some $P C N A$ reactivity is observed in the stalk (arrow) of the choroid plexus, whereas E2F5 reactivity, though weaker than at earlier stages, is detectable in the CPe. (G) E17.5 lateral ventricle choroid plexus stained for PCNA. No staining is observed in the choroid plexus. Cb, cerebellum; CPe, choroid plexus epithelium; CPm, choroid plexus mesenchyme; IV, IV ventricle; N, neuroepithelium. Scale bars: $250 \mu \mathrm{m}$ in A, $50 \mu \mathrm{m}$ in B,C, $200 \mu \mathrm{m}$ in E,F, G.
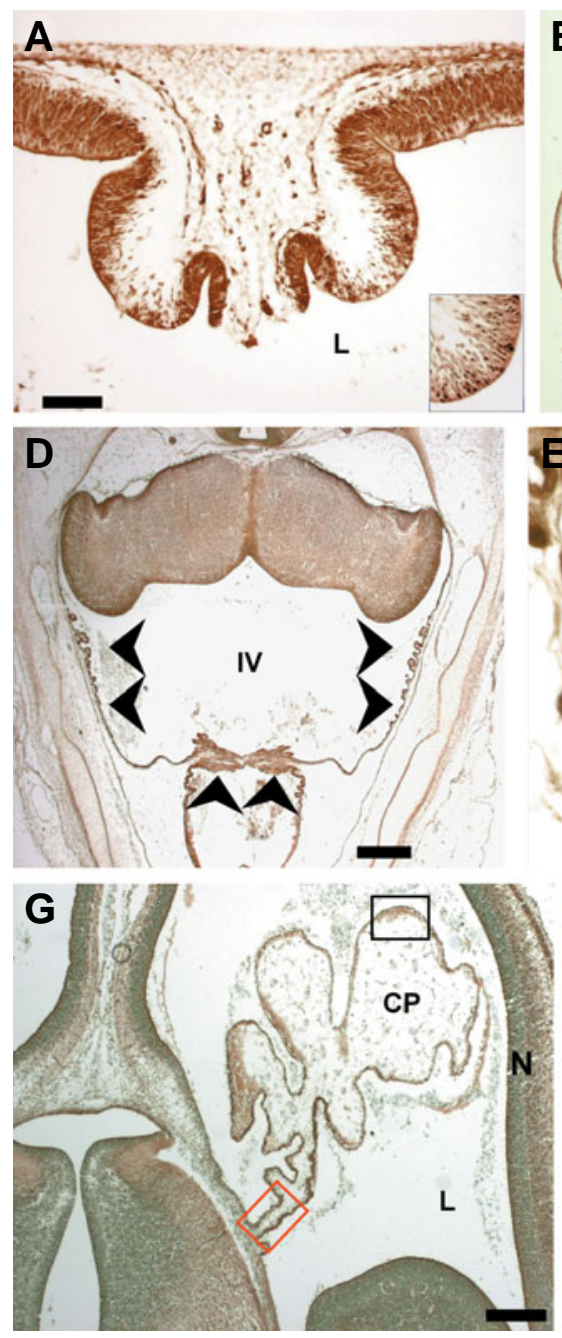
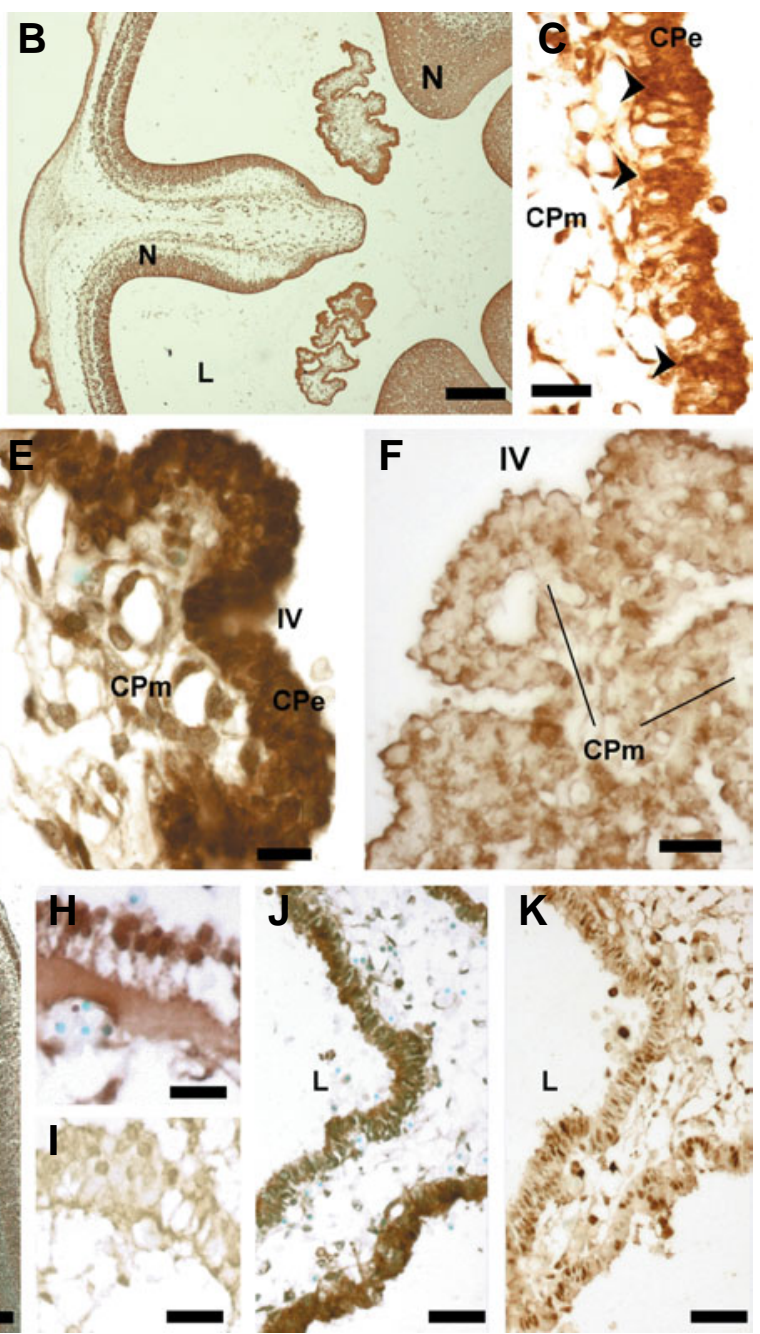

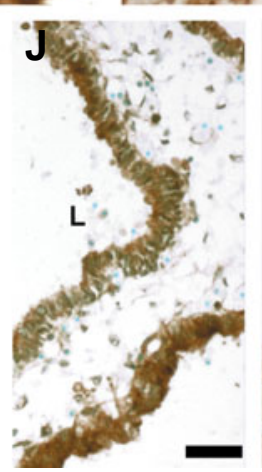

F

IV
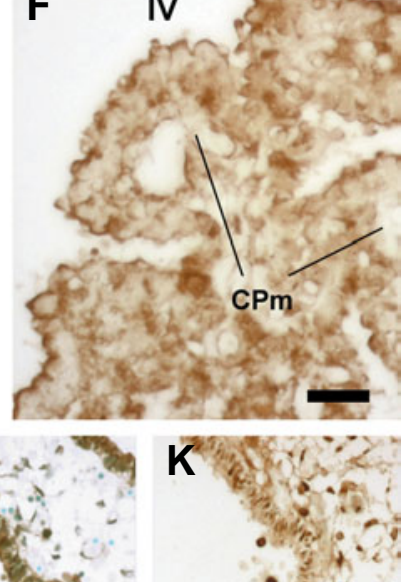
L

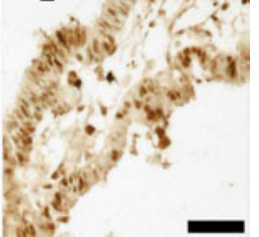

Fig. 4. E2F5 and PCNA protein expression in the human embryonic CPe. (A) Telencephalic vesicle of a human embryo at $44 d g$ stained for E2F5; the insert shows PCNA staining in an adjacent section. (B) Telencephalic vesicle of a human embryo at 52dg stained for E2F5. (C) Lateral ventricle choroid plexus from a 52dg embryo; strong E2F5 reactivity is observed in nuclei of the pseudostratified CPe (arrowheads), butsome staining is observed also in the cytoplasm (D) 8 week IVth ventricle; the arrowheads indicate the location of the IVth ventricle choroid plexus. (E) High magnification of the 8 week IVth ventricle choroid plexus shown in (D). (F) High magnification of a 11 week IVth ventricle choroid plexus. Note the reduction in E2F5 staining and its cytoplasmic localization as compared to 8 week choroid plexus. (G) Section through the telencephalon of a 8 week human embryo stained for E2F5. (H) High magnification of a region of the $\mathrm{CPe}$ corresponding to that indicated in the black square in (G). (I) Adjacent section to that shown in (H) stained for PCNA; no reactivity is observed. (J) E2F5 staining of the choroid plexus stalk (region indicated by the red square in G). (K) PCNA staining of the choroid plexus stalk. CPe, choroid plexus epithelium; CPm, choroid plexus mesenchyme; IV, IV ventricle; L, lateral ventricle; $N$, neuroepithelium. Scale bars: $100 \mu \mathrm{m}$ in $A$, $400 \mu \mathrm{m}$ in $B, 25 \mu \mathrm{m}$ in $C, F, 200 \mu \mathrm{m}$ in $G, 800$ $\mu \mathrm{m}$ in $D, 15 \mu \mathrm{m}$ in $E, H, I, 50 \mu \mathrm{m}$ in $\mathrm{J}, \mathrm{K}$. 
chyme was only weakly positive with occasional nuclear staining. At 8 weeks of gestation, both lateral and IV ventricle choroid plexus, as well as the neuroepithelium, were E2F5-positive (Fig. 4D,G) In the IVth ventricle, strong nuclear labelling was mostly present in the pseudostratified CPe cells (Fig. 4E). E2F5 was localized mainly in the nucleus in the monostratified columnar cells of the telencephalic $\mathrm{CPe}$ (Fig. 4G-H), whilst the staining in the pseudostratified cells at the stalk of the choroid plexus was both nuclear and cytoplasmic (Fig. 4J). In the 11 week foetus, E2F5 immunoreactivity in the IVth ventricle choroid plexus was much lower and rather cytoplasmic (Fig. 4F), whereas nuclear staining was still obvious in the retina (not shown).

At 44dg both E2F5 and PCNA were detected in the nuclei of ependyma and choroid plexus primordia (Fig. 4A). At 8 weeks of gestation E2F5 staining in the lateral $\mathrm{CPe}$ was localized to the nucleus and no PCNA expression was detected (Fig. 4G-I). In contrast, at the stalk of the choroid plexus, both strong PCNA and E2F5 reactivity were detected, but no obvious pattern of cell labelling overlap was apparent (Fig. 4J-K). Also in the IVth ventricle $\mathrm{CPe}$ of the 8 week embryo and in that of the 11 week foetus, E2F5 and PCNA labelling did not seem to co-localize (not shown).

\section{Discussion}

This study has shown that the transcripts for foxJ1, E2F5 and p73 are expressed throughout the developing IVth ventricle choroid plexus in the mouse and that the expression of E2F5 protein parallels that of the mRNA, indicating that its expression is controlled at the transcriptional level. Furthermore, this study shows that there is conservation of E2F5 protein expression between human and mouse choroid plexuses during development.

\section{E2F5 expression is developmentally regulated both in mouse and human $\mathrm{CPe}$}

The E2F5 protein is present in the choroid plexus and neuroepithelium as shown by immunohistochemistry and by Western blotting of mouse brain extracts at all stages of development studied, but its expression is hardly detectable in extracts of adult brain. Overall, the intensity of E2F5 staining in the CPe appears to be slightly down-regulated with development both in mouse and human, as supported also by a decrease in mRNA in the mouse. The decrease in E2F5 protein we observe in the developing mouse and human brains are consistent with previous in situ hybridization studies showing high levels of E2F5 mRNA in the early developing mouse nervous system (E9.5) and low levels at E17.5-19.5, when neuronal differentiation is much advanced (Dagnino et al., 1997). Furthermore, a decrease in E2F5 protein at late stages of gestation has been reported also in the rat cortex and in PC12 cells induced to undergo neuronal differentiation (Persengiev et al., 1999). Overall these results suggest that E2F5 expression is developmentally regulated in a similar fashion both in the developing brain and choroid plexus in different species and that, at least in mice, its expression is controlled at the mRNA level.

\section{Translocation of the E2F5 protein in the developing choroid plexus}

An interesting feature of E2F5 expression pattern in the $\mathrm{CPe}$ is the change in its subcellular localization observed both in developing mouse and human choroid plexuses in vivo and in CPe cells

\section{in vitro.}

At early stages of $\mathrm{CPe}$ development, the E2F5 protein is predominantly found in the nuclei. However, at later stages of gestation, when the epithelium from pseudostratified and columnar becomes cuboidal, like in the adult, E2F5 is mainly found in the cytoplasm (summarized in Fig. 5). Interestingly, in the pseudostratified choroid plexus stalk nuclear expression of E2F5 is maintained at late stages of development. Differences in subcellular localization are found also where both nuclear and cytoplasmic localization are clearly observed, with the latter appearing more frequently as the culture expands. Neither nuclear nor cytoplasmic expression of E2F5, however, correlates with the proliferative activity of the CPe cells in vivo. In the mouse IV ventricle $\mathrm{CPe}$, cell proliferation occurs mainly in the stalk region at all the stages examined, whereas in the lateral ventricle $\mathrm{CPe}$ PCNA-positive cells are scattered throughout the epithelium, rather than localized to the stalk, at early stages of development. At late stages of development PCNA reactivity becomes largely localized to the stalk also in the lateral ventricle. This suggests a difference in the growth mode of IV and lateral ventricle $\mathrm{CPe}$, with the former growing from its stalk and the latter initially growing also by intercalated cell division within the epithelium.

The biological significance of the changes in the subcellular localization of E2F5 in the CPe we have reported here has yet to be understood. Previous in vitro studies have shown that mouse embryonic fibroblasts from E2F5 knockout mice proliferate normally (Lindeman et al., 1998). Our study suggests that also in the CPe E2F5 is not involved in cell cycle control, as E2F5 translocation to different cell compartments does not coincide with changes in $\mathrm{CPe}$ cell proliferation in vivo. In other systems, however, cytoplasmic localization of E2F4/E2F5 has been associated with the proliferative state and translocation to the nucleus with an inhibitory effect on the cell cycle (Gaubatz et al., 2000; Muller et al., 1997). Overall the changes in subcellular localization we
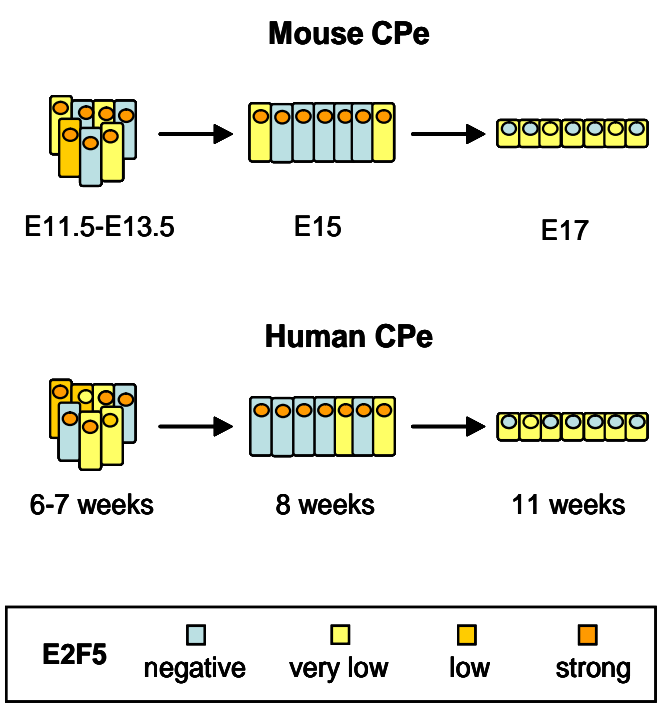

Fig. 5. Diagram summarizing changes in E2F5 protein expression with maturation of the $\mathrm{CPe}$ in mouse and human developing $\mathrm{CPe}$. The stages of $\mathrm{CPe}$ development, pseudostratified, columnar and cuboidal and their approximate timing in mouse (E, embryonic day) and human (weeks, weeks of gestation) are shown. Similar patterns are observed in different ventricles and in human and mouse CPe. 
observe appear to correlate with maturation of the $\mathrm{CPe}$, as indicated by its morphological changes, possibly reflecting the functional state of $\mathrm{CPe}$ cells.

\section{Conclusions}

E2F5 protein expression in the developing choroid plexus appears to be conserved across species supporting the view that it plays an important role in the development and function of this organ. Its dynamic expression suggests a role in the maturation process of $\mathrm{CPe}$ cells, consistent with the defective function of the $\mathrm{CPe}$ leading to hydrocephalus in E2F5 knockout mice. Therefore, the future challenge will be to establish the precise role of this gene in $\mathrm{CPe}$ development and whether it is on the same/share pathways with other genes expressed in the $\mathrm{CPe}$, such as $\mathrm{p73}$, whose inactivation also results in a hydrocephalic phenotype. It will be also important to investigate whether mutations in any of these genes are present in patients with non obstructive hydrocephalus.

\section{Materials and Methods}

\section{Tissues and cells}

Out-bred CD-1 albino mice supplied by Charles River Mouse Farms (Kent, UK) and housed under Home Office regulations were used. The morning of detection of a vaginal plug was designated as embryonic day 0.5 (E0.5). The uterus containing between 9 and 21 embryos was removed whole and placed in ice-cold phosphate-buffered saline (PBS). The embryos were freed from uterine and amniotic tissues and decapitated. The head was either fixed immediately for immunohistochemistry or the IV ventricle choroid plexus was dissected on ice for protein or mRNA analysis. For RT-PCR analysis the IV ventricle choroid plexus from adult mice was also dissected.

Human embryos between 40 days and 11 weeks of gestation were obtained under ethical approval from the Wellcome/MRC-funded Human Developmental Biology Resource.

The rat CPe cell line, TRCSFB-2 (courtesy of Tetsuya Terasaki, Tokohu University, Japan), was grown in DMEM containing $10 \%$ foetal calf serum at $33^{\circ} \mathrm{C}$ on $13 \mathrm{~mm}$ coverslips and transferred at $37^{\circ} \mathrm{C} 48$ hours before being processed for immunocytochemistry.

\section{Immunohistochemistry}

The following primary antibodies were used: E2F5 rabbit polyclonal antibody (clone E19, Santa Cruz Biotechnology, Inc., California, USA; 1:50); anti-proliferating cell nuclear antigen (PCNA) monoclonal antibody (clone PC10, Santa Cruz Biotechnology, Inc., California, USA; 1:100). Cy3-conjugated AffiniPure goat anti-mouse IgG immunoglobulins (Jakson Immunoresearch Laboratories, Inc,West Grove, USA; 1:50), biotin conjugated goat-anti-mouse and goat-anti-rabbit goat immunoglobulins (DAKO,UK; 1:100) and StreptABComplex/HRP (ABC duet kit, DAKO Ltd, UK; 1:100) were used for primary antibody detection.

Six micrometer thick paraffin sections were deparaffinized and either heated in a microwave at $540 \mathrm{~W}$ in $0.01 \mathrm{M}$ citric acid $(\mathrm{pH} 6.0)$ for 5 to 10 minutes or incubated 2 minutes at $37^{\circ} \mathrm{C}$ with 4 units $/ \mathrm{ml}$ of a non-specific type XIV protease from'Streptomyces griseus (Sigma, UK) for epitope unmasking. Immunhistochemistry on sections and cultured cells was carried out essentially as previously described (O'Neill et al., 2004; Reid and Ferretti, 2003). When fluorescent detection was used, nuclei were counterstained with bisbenzimide $\mathrm{H} 33258$ fluorochrome (Hoechst nuclear stain). Negative controls, where the primary antibody was omitted were run in all experiments. In the case of E2F5 antibody, staining was blocked by pre-adsorbing the antibody with the E2F5 blocking peptide corresponding to the amino-terminus of human E2F5 (Santa-Cruz, USA; 1:10) for 1 hour at $37^{\circ} \mathrm{C}$. Tissues and cells were viewed under a Zeiss axioplan microscope and digitally scanned using a Hamamatsu digital camera (C4742-95, Hamamatsu Photonics KK, Japan) into Openlab software (Improvision Ltd, UK).

\section{$R T-P C R$}

RT-PCR was carried out essentially as previously described (Zhang et al., 2002). IVth ventricle choroid plexus were dissected from embryos at different developmental stages and stored in $1 \mathrm{ml} \mathrm{TRI-Reagent} \mathrm{(Sigma-}$ Aldrich, Dorset, UK) at $-20^{\circ} \mathrm{C}$ until RNA extraction according to the manufacturer instructions. PCR of RNA samples was routinely carried out with the same primers used to amplify the CDNA obtained by RT to rule out genomic DNA contaminations. cDNA was synthesised using the $\mathrm{M}$ MLV reverse transcriptase (Promega, Southampton, UK) from $1 \mu \mathrm{g}$ of RNA using random hexamers (Promega, UK). The PCR step was performed using Taq polymerase (Promega) at an annealing temperature of $57^{\circ} \mathrm{C} ; 30$ cycles were used for all primers. The sequence and expected product size of the primer pairs used are:

GAPDH (491bp): For: 5'-TTCCAGTATGACTCCACTCACG-3', Rev: 5'-GGATGCAGGGATGATGTTCT-3';

E2F5 (227 bp):

For: 5'-TGTGGCTACAGCAAAGCATC-3', Rev: 5'-GGCCCTGAGTGACTCTTCAG-3';

FoxJ1 (205 bp):

For: 5'-TACTGCTGACCCAGGAGGAG-3', Rev:5- GGTAGCAGGGCAGTTGATGT-3', Transthyretin (505 bp):

For: 5'-CAGATCCACAAGCTCCTGAC-3',

p73 (232 bp) Rev: 5'-CTGCTTTGGCAAGATCCTGC-3';

\section{For: 5'-AGAGTGTGGTTGTGCCGTATG-3', Rev: 5'-TCCCGGTAATGGTCTTCATC-3'.}

PCR products were visualized on a $1.5 \%$ agarose gel containing ethidium bromide under ultraviolet light and imaged by using a Gel imaging system.

\section{Western blotting}

Embryonic brains collected at different developmental stage were stored at $-20^{\circ} \mathrm{C}$ in RIPA buffer $(150 \mathrm{NaCl}, 1.0 \%$ IGE-PAL CA630 NP-40, $0.5 \%$ deoxycholate, $0.1 \%$ sodium dodecyl sulphate (SDS) and $50 \mathrm{mM}$ Tris buffer) containing $60 \mu \mathrm{l} / \mathrm{ml}$ of a protease cocktail inhibitor (Sigma, UK). Upon thawing the brains were homogenized with a Dounce homogenizer at $4^{\circ} \mathrm{C}$. Total protein concentration was quantified using the bicinchroninic acid (BCA) Protein Assay kit (Pierce). Sixty $\mu \mathrm{g}$ of protein/ lane were separated by $12 \%$ SDS-PAGE and gels were blotted onto PVDF (polyvinylidene difluoride) membranes (Amersham Biosciences) by semi-dry blot transfer and processed for immunodetection. Immunoreactivity was detected by enhanced chemical luminescence $(E C L)$.

\section{Acknowledgements}

We wish to thank the Wellcome/MRC-funded Human Developmental Biology Resource for providing the embryonic human material. This work was supported by the Child Research Appeal Trust.

\section{References}

CATALA, M. (1998). Embryonic and fetal development of structures associated with the cerebro-spinal fluid in man and other species. Part I: The ventricular system, meninges and choroid plexuses. Arch Anat Cytol Pathol 46: 153-69.

CHEN, J., KNOWLES, H. J., HEBERT, J. L. and HACKETT, B. P. (1998). Mutation of the mouse hepatocyte nuclear factor/forkhead homologue 4 gene results in an absence of cilia and random left-right asymmetry. J Clin Invest 102: 107782. 
COULY, G. F., COLTEY, P. M. and LE DOUARIN, N. M. (1993). The triple origin of skull in higher vertebrates: a study in quail-chick chimeras. Development 117: 409-29.

DAGNINO, L., FRY, C. J., BARTLEY, S. M., FARNHAM, P., GALLIE, B. L. and PHILLIPS, R. A. (1997). Expression patterns of the E2F family of transcription factors during mouse nervous system development. Mech Dev 66: 13-25.

GAUBATZ, S., LINDEMAN, G. J., ISHIDA, S., JAKOI, L., NEVINS, J. R., LIVINGSTON, D. M. and REMPEL, R. E. (2000). E2F4 and E2F5 play an essential role in pocket protein-mediated G1 control. Mol Cell 6: 729-35.

KUME, T., DENG, K. Y., WINFREY, V., GOULD, D. B., WALTER, M. A. and HOGAN, B. L. (1998). The forkhead/winged helix gene Mf1 is disrupted in the pleiotropic mouse mutation congenital hydrocephalus. Cel/ 93: 985-96.

LINDEMAN, G. J., DAGNINO, L., GAUBATZ, S., XU, Y., BRONSON, R. T., WARREN, H. B. and LIVINGSTON, D. M. (1998). A specific, nonproliferative role for E2F-5 in choroid plexus function revealed by gene targeting. Genes Dev 12: $1092-8$

MUller, H., MORONI, M. C., VIGO, E., PETERSEN, B. O., BARTEK, J. and HELIN, K. (1997). Induction of S-phase entry by E2F transcription factors depends on their nuclear localization. Mol Cell Biol 17: 5508-20.

O'NEILL, P., WHALLEY, K. and FERRETTI, P. (2004). Nogo and Nogo-66 receptor in human and chick: Implications for development and regeneration. Dev. Dyn. 231: 109-21.

PERSENGIEV, S. P., KONDOVA, II and KILPATRICK, D. L. (1999). E2F4 actively promotes the initiation and maintenance of nerve growth factor-induced cell differentiation. Mol Cell Biol 19: 6048-56.

REID, S. and FERRETTI, P. (2003). Differential expression of FGFRs in the developing murine choroid plexus. Dev Brain Res 141: 15-24.

SHUANGSHOTI, S. and NETSKY, M. G. (1966). Histogenesis of choroid plexus in man. Am J Anat 118: 283-316.

VAISHNAV, Y. N., VAISHNAV, M. Y. and PANT, V. (1998). The molecular and functional characterization of E2F-5 transcription factor. Biochem Biophys Res Commun 242: 586-92.

WILTING, J. and CHRIST, B. (1989). An experimental and ultrastructural study on the development of the avian choroid plexus. Cell Tissue Res 255: 487-94.

YANG, A., WALKER, N., BRONSON, R., KAGHAD, M., OOSTERWEGEL, M., BONNIN, J., VAGNER, C., BONNET, H., DIKKES, P., SHARPE, A., MCKEON, F. and CAPUT, D. (2000). p73-deficient mice have neurological, pheromonal and inflammatory defects but lack spontaneous tumours. Nature 404: 99-103.

ZHANG, F., CLARKE, J. D. W., SANTOS-RUIZ, L. and FERRETTI, P. (2002). Differential regulation of FGFRs in the regenerating amphibian spinal cord in vivo. Neuroscience 114: 837-48.

ZHENG, X. and CHEN, X. (2001). Aquaporin 3, a glycerol and water transporter, is regulated by $\mathrm{p} 73$ of the $\mathrm{p} 53$ family. FEBS Lett 489: 4-7.

Received: March 2005 Reviewed by Referees: March 2005

Modified by Authors and Accepted for Publication: April 2005 